\title{
Possibility for Recognition of Psychic Brain Activity with Continuous Wavelet Analysis of EEG
}

\author{
Evgeny A. Yumatov', Alexandr E. Hramov², Vadim V. Grubov², Oleg S. Glazachev³, \\ Elena N. Dudnik ${ }^{3}$, Nikolay A. Karatygin ${ }^{1}$
}

${ }^{1}$ P.K. Anokhin Research Institute of Normal Physiology, Moscow, Russia

${ }^{2}$ Yuri Gagarin State Technical University of Saratov, Saratov, Russia

${ }^{3}$ First Moscow State Medical University of the Ministry of Health of the Russian Federation (Sechenov University), Moscow, Russia

Email: eayumatov@mail.ru,hramovae@sstu.ru,vvgrubov@gmail.com,glazachev@mail.ru,nikolaseve@yandex.ru,elenad72@list.ru

How to cite this paper: Yumatov, E.A., Hramov, A.E., Grubov, V.V., Glazachev, O.S., Dudnik, E.N. and Karatygin, N.A. (2019) Possibility for Recognition of Psychic Brain Activity with Continuous Wavelet Analysis of EEG. Journal of Behavioral and Brain Science, 9, 67-77.

https://doi.org/10.4236/jbbs.2019.93006

Received: January 31, 2019

Accepted: February 26, 2019

Published: March 1, 2019

Copyright $\odot 2019$ by author(s) and Scientific Research Publishing Inc. This work is licensed under the Creative Commons Attribution International License (CC BY 4.0).

http://creativecommons.org/licenses/by/4.0/

\begin{abstract}
The brain is a unique organization in nature, possessing the ability for psychic activity, which manifests itself in thoughts, feelings and emotions. In present days, numbers of mathematical methods for analysis of electroencephalogram (EEG) were developed with continuous wavelet transform being one of the most successive approaches for studying of brain activity. This paper is aimed to develop methods for investigation of psychic brain activity with help of continuous wavelet analysis of EEG. Ability of human to realize semantic content of the image presented on the screen was tested. Experiment was accompanied with simultaneous EEG recording, which was held with developed software and PC-based experimental setup. The information capabilities of continuous wavelet transform-based method for EEG analysis were improved for the recognition of specific patterns in human brain activity. Comparative wavelet analysis was carried out for EEG recordings at the moment of awareness of the semantic content of the image and for EEG recordings in the absence of conscious (subjective) perception of the semantic content of the image. Significant differences were shown in the alpha rhythm of EEG for the moments of awareness of the semantic content of the image and for the moments of absence of conscious perception. Continuous wavelet analysis of EEG showed that the alpha rhythm is the main EEG rhythm, which can be used to estimate the presence of subjective perception of the visual image. Significant differences were shown in the alpha rhythm of EEG for the moments of awareness of the semantic content of the image and for the moments of absence of conscious perception. Conducted studies allow to conclude that revealing of brain activity related to visual image awareness is possible through analysis of EEG.
\end{abstract}




\section{Keywords}

Brain, Psychic, Consciousness, Electroencephalogram, Continuous Wavelet Analysis

\section{Introduction}

The brain is a unique organization in the living nature, possessing the ability for psychic activity, which manifests itself in thoughts, feelings, emotions, i.e. in subjective human's perception of himself and surrounding world.

Despite of substantial progress in modern psychophysiology and neurophysiology, the existing methods cannot allow direct registration and investigation of patterns of brain psychic activity [1] [2] [3] [4]. Using of classic methods for analysis of electroencephalogram (EEG) reveals nonstationary nature of EEG rhythmic activity along with high variability of forms and frequency ranges, which greatly reduce informational capabilities of traditional methods for spectral signal analysis.

Development of conceptually new informational methods capable of deciphering psychic activity patterns in neurophysiological processes is crucial for revealing the nature of brain psychic activity. In present days, numbers of modern mathematical methods for analysis of electroencephalogram (EEG) were developed. One of the most successive approaches is continuous wavelet transform (CWT) [5] [6] [7] [8] [9].

Continuous wavelet transform's main advantage over the classic spectral analysis method is the capability of time-frequency analysis of complex nonstationary signals such as EEG. At the same time, possibility for detection of psychic brain activity with help of EEG analysis is still questionable.

The main aim of the present research is to develop informational technology and methods for studying psychic brain activity with help of continuous wavelet analysis of EEG signals.

The objectives of the research are:

1) Improvement of informational capabilities of continuous wavelet transform-based method for EEG analysis, designing of specialized wavelet basis for increasing the effectiveness of detection of specific psychic activity patterns in human brain.

2) Testing of temporal visual discriminating ability of human to realize semantic content of the image during short presentation time.

3) Registration of EEG in different areas of brain, including visual cortex, during the testing of individual discriminating ability of human to realize semantic content of image.

4) Comparative wavelet analysis for EEG recordings at the moment of awareness of the semantic content of the image and for EEG recordings in the absence of conscious (subjective) perception of the semantic content of the image. 


\section{Materials and Methods}

In the present study experimental test is performed to evaluate the ability of human to realize semantic content of demonstrated image during very short presentation time. Design of the experiment suggests demonstration of series of images to the human subject. Presentation time is shortened with each subsequent image: the first image is presented for the longest time period while presentation time for the last image is close to 0 . The main point of the test is to find the threshold point, when human could recognize the previous image, but fails to recognize the next one due to the short presentation time [10] [11] [12].

Experimental research is approved on the meeting of local ethic committee of First Moscow State Medical University of the Ministry of Health of the Russian Federation (Sechenov University) on May 18, 2014. All experimental works are held in agreement with ethical standards laid down in the 1964 Declaration of Helsinki. All persons gave their informed consent prior to their inclusion in the study, to personal and experimental data processing.

The study uses PC-based experimental setup along with software for testing and detection of two types of brain activity based on EEG properties. The first type of activity is related to subjective conscious perception of visual image and the second one is related to absence of this perception due to the short-term presentation of image [13].

Experimental setup is based on PC with two displays, which are used simultaneously during the testing. The first display is used by the experimenter and shows information about experimental settings such as number and type of presented images, time of presentation, etc. The software also provides "Experimenter's window" on this display with instruments to adjust mentioned parameters according to desired experimental design. The second display is used for presentation of visual stimuli to the human subject in "Presentation window". This window contains consequently demonstrated images with presentation time reduced on each consequent step.

The task of the subject is to carefully observe the second display with "Presentation window" and to try to realize demonstrated images. At some threshold point the subject sees image shortly but fails to realize and recognize it. After the experiment the subject is asked for how many images he/she recognized and which images he/she didn't recognize due to short presentation. This is used to estimate the threshold point.

Experimental setup also includes means for EEG recording. Electroencephalograph "Neuron-Spectrum" (Ivanovo, Russia) is used to record EEG signals from 10 electrodes in occipital $(\mathrm{O} 2, \mathrm{O} 1)$, parietal (P4, P3), central (C4, C3), frontal (F4, F3) and temporal (T4, T3) areas. These electrodes are placed according to standard monopolar " 10 - 20" scheme. Reference electrodes are attached to earlobes. Recorded EEG signals are filtered by band-pass filter with cut-off points at 0.5 and $75 \mathrm{~Hz}$ and by $50 \mathrm{~Hz}$ notch filter. Sample rate of recorded EEG signals is $200 \mathrm{~Hz}$. 
EEG recording is held for 10 healthy students-men in age 18 - 22 years and employees in age 45 - 60 years of the First Moscow State Medical University of the Ministry of Health of the Russian Federation (Sechenov University) with normal or restored to normal visual perception.

Each experiment has two stages: with closed eyes and open eyes. The first stage with closed eyes is a standard practice to obtain baseline EEG for further analysis. The second stage with open eyes is an active part of experiment. During this stage subject observes 10 sequences of images with 25 images in each. Sequences are demonstrated with time intervals of few minutes.

Developed software is based on principles from [14] [15]. The main purposes of the software are to prepare series of images for demonstration, to demonstrate subsequent images with subsequently reduced time of presentation and to form journal of the experiment.

The software holds database with 68 different easily recognized images of animals, plants, everyday devices, furniture, etc. All images are colored and have sufficient resolution ( $300 \mathrm{dpi})$.

The software allows to arrange the images from database in random order to form sequences for presentation during the experiment. This allows to prevent adaptation of the subject to the task and to avoid stereotypic answers.

Images from constructed sequences can be presented at different rate. The software has options to choose number of images in sequences, time intervals for each presentation and for pauses between subsequent presentations. Duration of image presentation is affected by refresh rate of display. For example, for display with refresh rate $f$ of $60 \mathrm{~Hz}$ minimal time of presentation is $1 / f=17 \mathrm{~ms}$. For this display images will be presented for 17 -fold time intervals $(17,34,51 \ldots \mathrm{ms})$ according to the chosen experimental design.

The software also features options for choosing algorithms of presentation time decrease. Experimenter can choose between two algorithms: 1) each subsequent presentation time is two times shorter than previous, 2) each subsequent presentation time is shortened by some constant value.

Duration of pauses between two subsequent presentations can also be chosen and commonly equals to several seconds. During this time interval the subject observes blank screen. Such pauses are aimed to prevent the influence of previously demonstrated images on perception of current image.

The software automatically creates journal file that contains all information about the experiment. This data can be used in further analysis of EEG signals.

The software provides options to change brightness, clearness, saturation of presented images which allow to make the particular image more/less recognizable. Such technique for changing informational properties allows to detect two different states in subjects: conscious perception of visual image and absence of this perception. In proposed experimental procedure developed setup provides opportunity to find the moment when subjective recognition occurs for image with short exposition time. 


\section{Calculations}

Continuous wavelet transform (CWT) is used for analysis of obtained EEG data [5] [6]. CWT is known to be effective in time-frequency analysis of nonstationary signals (including EEG) and as basis for development of algorithms for automatic detection of specific EEG patterns [7] [8] [9].

CWT is a convolution of analyzed signal $x(t)$ and set of basic functions $\varphi_{s, \tau}$ :

$$
W(s, \tau)=\int_{-\infty}^{+\infty} x(t) \varphi_{s, \tau}(t) d t
$$

where the asterisk denotes the complex conjugation. Each basic function $\varphi_{s, \tau}$ from this set is obtained by compression/extension and time shift of one function called mother wavelet as following:

$$
\varphi_{s, \tau}(t)=\frac{1}{\sqrt{s}} \varphi_{0}\left(\frac{t-\tau}{s}\right),
$$

where $s$-time scale responsible for compression or extension of mother wavelet, $\tau$-time shift of wavelet function, $\varphi_{0}$-mother wavelet. Instead of time shift $s$ it is possible to introduce frequency $f=1 / s$, which is more convenient in terms of time-frequency analysis.

The CWT-based spectral analysis of biological signals including EEG is usually performed with the Morlet wavelet as the mother function [16] since Morlet wavelet provides good resolution both in time and frequency domains:

$$
\varphi(t)=\pi^{-0.25} e^{j w_{0} t} e^{-\frac{\tau^{2}}{2}}
$$

The Morlet wavelet represents the complex harmonic oscillating function, $e^{j w_{0} t}$, modulated by the Gaussian function, $e^{-\frac{\tau^{2}}{2}}$. The parameter $\omega_{0}$ is central frequency of Morlet wavelet, it is usually chosen to be $\omega_{0}=2 \pi$ as such value leads to the simple correlation between frequency and time scale: $f=1 / \mathrm{s}$. Morlet wavelet with chosen parameters provides means to analyze short fragments of data series (3 - 5 oscillations) and, therefore, can be used for analysis of different types of EEG patterns.

The most common part of CWT-based signal analysis is investigation of wavelet energy spectrum $E\left(f_{s}, t\right)$ which provides information about time-frequency structure of signal.

$$
E\left(f_{s}, t\right)=\left|W\left(f_{s}, t\right)\right|^{2}
$$

Wavelet energy spectrum $E\left(f_{s}, t\right)$ can be averaged over some specific frequency range in order to analyze time dynamics in this range.

For more detailed analysis of frequency dynamics in signal at given time moment $t_{0}$ momentary wavelet energy distribution $E_{t_{0}}$ is used:

$$
E_{t_{0}}\left(f_{s}\right)=\left|W\left(f_{s}, t=t_{0}\right)\right|^{2}
$$

Investigation of main frequencies in signal can be performed with another CWT-based technique-construction of skeletons. Skeletons of wavelet surface 
are obtained by picking few local maxima from momentary wavelet energy distribution $E_{t_{0}}$ and repeating this process for each time moment in the signal. Resulting skeletons can be used for tracking most significant frequency components in the signal and for analyzing their time dynamics. Such technique provides information about appearance/disappearance of certain specific rhythmic components, which can help to investigate shifts in subject's perception.

Since the main aim of the present paper is to distinct two types of brain activity on EEG (conscious perception of visual image and absence of this perception) Wilcoxon signed-rank test is used. Wilcoxon signed-rank test is a non-parametric statistical hypothesis test used to compare two related samples to assess whether their population mean ranks differ [17].

\section{Results}

During experimental work EEG signals are recorded for 10 subjects in the age of 18 - 45. Each subject observes 10 series of 25 images with subsequently shortened demonstration time. Example of EEG recordings is illustrated on Figure 1. Figure 1 shows EEG signals for 10 channels (O2, O1, P4, P3, C4, C3, F4, F3, T4, T3) with special marks for start and end of demonstration of each image. Thus, EEG episodes during demonstrations of images and between demonstrations can be analyzed in order to test human's visual discrimination ability to realize content of observed image.

EEG signals are investigated with CWT-based methods. For each channel from EEG recording wavelet spectrum, skeleton and averaged wavelet energy distribution are constructed (see Figure 2). Figure 2(a) shows initial EEG signal from one channel (O1) during one series of image demonstration with dark blue vertical lines that mark the start and the end of each image's demonstration. Two specific EEG episodes are marked on Figure 2(a) as examples: the first one where subject recognizes presented images well (marked with light blue frame) and the second one where subject fails to recognize images (marked with light green frame). Figures 2(b)-(g) demonstrate results of time-frequency analysis of these two episodes with wavelet-based methods: wavelet spectrums (Figure 2(b), Figure $2(\mathrm{c})$ ), distribution of wavelet energy averaged over alpha frequency range (Figure 2(d), Figure 2(e)), skeletons (Figure 2(f), Figure 2(g)).

Distributions of wavelet energy (wavelet spectrum) are demonstrated in the lower part of Figure 2(b) and Figure 2(c) alongside with the initial EEG signals. Wavelet spectrum as projection of surface of wavelet energy distribution on " $t$ - $f$ " plane provides general information about time-frequency structure of EEG signal. As seen from Figure 2(b) and Figure 2(c) there are several dominant frequency components on the signal: $2-5 \mathrm{~Hz}$ component that corresponds to delta frequency range, 8 - $14 \mathrm{~Hz}$ - to alpha activity and $15-30 \mathrm{~Hz}-$ to beta activity.

It is known that activity in these frequency ranges and transitions of dominant frequencies from one range to another before, during and after demonstration of image can provide information about human's attention and concentration [18]. 


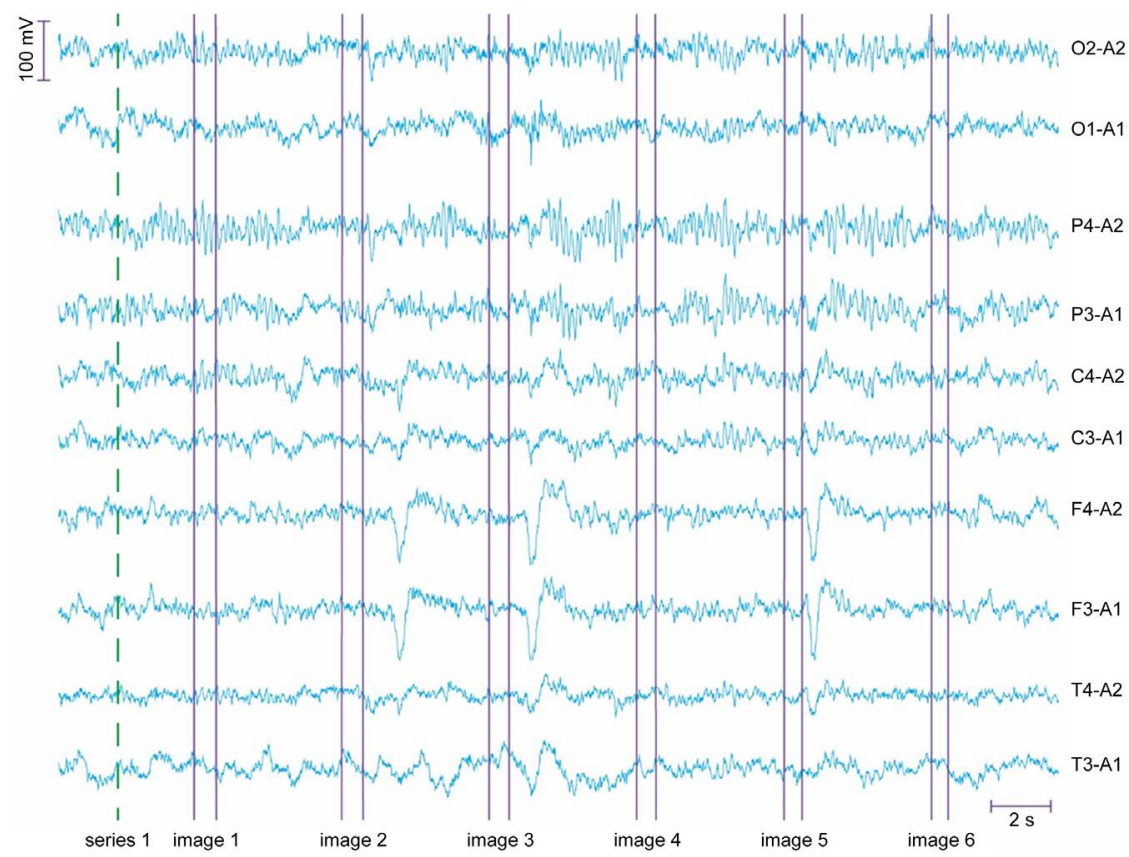

Figure 1. Example of EEG recording for 10 channels during perception of visual stimuli: green dashed marker corresponds to the start of series; dark blue solid markers correspond to the start and the end of presentation of each image in series.
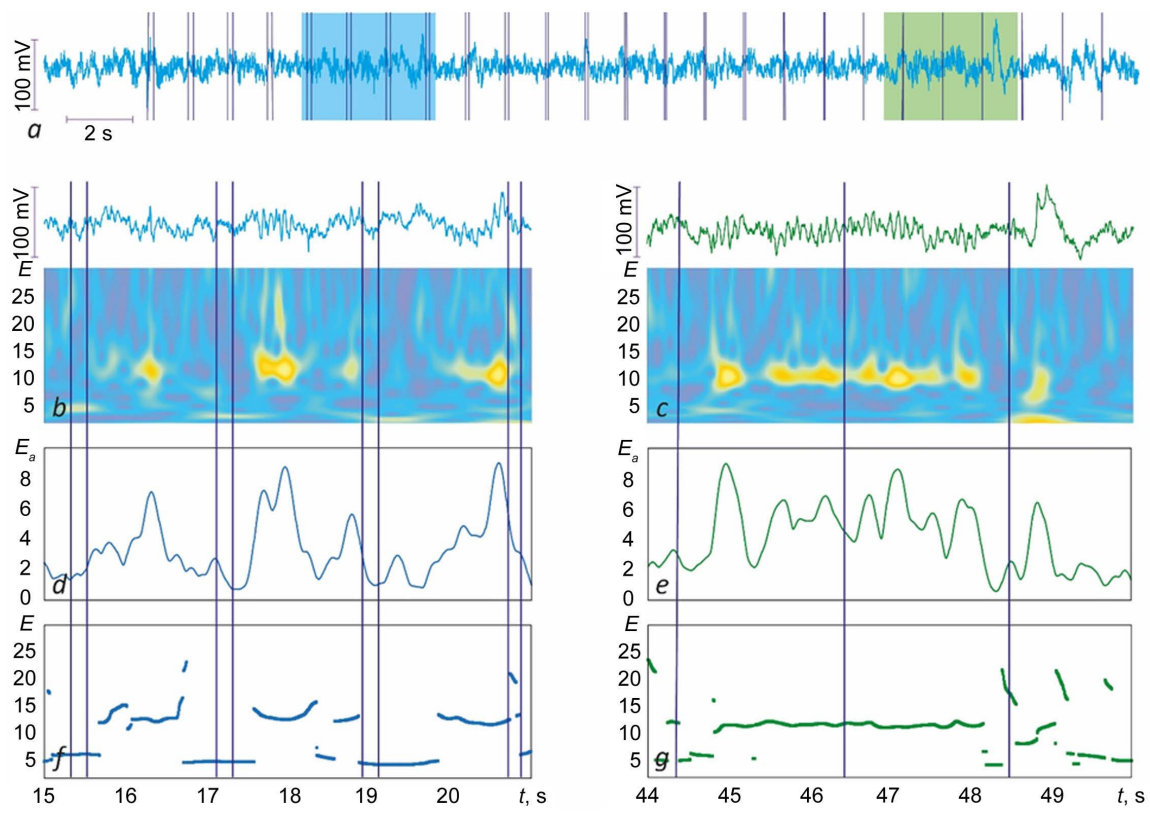

Figure 2. Results of EEG time-frequency analysis: (a) initial EEG signal; dark blue vertical lines mark the start and the end of each image's demonstration, light blue and light green frames correspond to EEG episodes with and without recognition of images; (b, c) EEG episodes and wavelet spectrums for cases with and without recognition of images; (d, e) averaged wavelet energy distributions for cases with and without recognition of images; $(f, g)$ skeletons of wavelet surface for cases with and without recognition of images.

From Figure 2(b), Figure 2(c) one can clearly see that alpha rhythm appears and disappears through EEG recording, which is assumed to be connected with 
human's ability to realize content of observed image [19] [20]. Thus, distribution of wavelet energy averaged over alpha frequency range (see Figure 2(d), Figure $2(\mathrm{e})$ ) can be considered in order to provide more detailed analysis in alpha range.

Figure 2(d) and Figure 2(e) demonstrate that alpha rhythm has number of high-energy spikes with certain drops between them. Such shifts correspond to moments of appearance and disappearance of alpha rhythm. For more clear representation averaged wavelet energy is accompanied with skeletons of wavelet surface (see Figure 2(f), Figure 2(g)). Skeleton provides information whether or not alpha rhythm is the dominant frequency at given moment and when it loses its dominant status.

From Figures 2(d)-(g) one can see that spikes of alpha activity mostly correspond to time moments before and after demonstration of image and alpha rhythm is the dominant EEG frequency at these moments. At the same time, alpha rhythm activity almost completely disappears (drop in average wavelet energy distribution and dominant EEG frequency component changes to delta or beta) during perception of images with long demonstration time (200 - $300 \mathrm{~ms})$.

Analysis shows that in the case of images with short demonstration time $(\sim 0$ ms) decrease and subsequent increase is far less significant. For example, the second EEG fragment (Figure 2(c), Figure 2(e), Figure 2(g)) shows that alpha activity suffers no substantial decrease or increase for images with very short demonstration time.

Thus, all image demonstrations can be divided into two groups: 1) with increase in alpha activity before and after demonstration and considerable decrease during demonstration (case of Figure 2(b), Figure 2(d), Figure 2(f)); 2) without described dynamics in alpha frequency range (case of Figure 2(c), Figure 2(e), Figure 2(g)). It is assumed that dynamics of the first type corresponds to cases when the subject is possible to realize semantic content of the image while dynamics of the second type reflects the absence of conscious (subjective) perception.

After each series of image demonstration, the subject is asked to pick the time moment $t_{c r}$ when he/she recognizes the previous image and doesn't recognize the next image. This time moment can serve as a possible milestone that separates the state with ability to subjective perception from the state without this ability.

In each series image demonstrations are divided into two groups (two related samples): before moment $t_{c r}$ (possible state of subjective perception) and after moment $t_{c r}$ (possible state with lost ability for subject perception). For these two groups statistical analysis is carried out with use of Wilcoxon signed-rank test. In this analysis the intensity of alpha activity during image perception is compared for these two samples. Wilcoxon criterion is calculated and appears to be $P<<0.05$. This result implies significant difference in intensity of alpha rhythm in the state with conscious perception and the state without it.

\section{Discussion}

Having summarized the results of the experiments described above, one can see 
that the ability of human to recognize the semantic content of visual stimuli can vary during experiment according to demonstration time of stimuli. These differences in perception can be noted by directly asking the subject and by changes in time-frequency structure of corresponding EEG signals.

It is known that alpha rhythm in occipital area can provide information about human's attention and concentration [18]. Acquired results further contribute to understanding of relations between alpha activity and visual perception. The results show that EEG activity in alpha frequency range is connected to psychic activity during recognition of the semantic content of the image.

The most important thing is dynamics in alpha range (i.e. appearance and disappearance of alpha rhythm) before, during and after visual stimulus. Analysis reveals two scenarios of alpha rhythm dynamics: 1) in the state with conscious perception alpha activity is more pronounced before and after visual stimulus and demonstrate significant drop during perception of visual stimulus; 2) in the state without conscious perception dynamics of alpha rhythm is far less pronounced or completely absent.

The first of observed scenarios is commonly known in visual stimuli-based experiments [19] [20], while the second scenario is more interesting. Absence of significant changes in alpha activity may imply that the subject loses its ability for conscious perception. The second scenario mostly appears during perception of stimuli after time moment $t_{c r}$ i.e. when the subject fails to recognize the images with short demonstration time. This may suggest that the brain doesn't have enough time to process and recognize incoming visual stimulus, which, in its turn, leads to no increase in visual awareness and no drop in alpha activity.

While alpha activity dynamics is far less pronounced in the second scenario, it is not completely absent in most cases. Occasionally dynamics in alpha range can be observed even during image demonstration after moment $t_{c r}$ It can be assumed that less pronounced but still presented dynamics of alpha rhythms reflects some kind of psychic activity corresponding to human's self-appraisal about inability to recognize the semantic content of the image.

\section{Conclusions}

In the paper, new experimental setup is developed for testing human's ability to realize semantic content of the image during various presentation times. Numbers of experiments are held for volunteers with this setup in order to acquire multichannel EEG recordings.

CWT-based approach is used for time-frequency analysis of the obtained EEG signals. Analysis shows that human's ability to subjective perception of image can be judged according to the state and dynamics of alpha activity in EEG signal.

Significant differences are found in dynamics of alpha rhythm for case with conscious perception of image's semantic content and for case without this perception. 
Obtained results suppose that it is possible to detect psychic brain activity connected to perception of image.

Future studies can be aimed to more detailed CWT-based analysis of big experimental EEG data in order to find specific components of alpha rhythm, which are characteristic for psychic brain activity.

\section{Fund}

This work is supported by RFBR (grant 19-07-00008\19) and by the Council of the President of the Russian Federation for Support of Young Scientists and Leading Scientific Schools (Grant MK-3305.2017.2).

\section{Conflicts of Interest}

The authors declare no conflicts of interest regarding the publication of this paper.

\section{References}

[1] Popper, K. (2008) Knowledge and Psychophysical Problem: In Defense of the Interaction. Zhuravlev I.V., Eds., Translate from English, Moscow, 256 p.

[2] Yumatov, E.A. (2014) To Knowledge of the Origin of the Brain Mental Activity. World Journal of Neuroscience, 4, 170-182. https://doi.org/10.4236/wjns.2014.42020

[3] Yumatov, E.A. (2014) The Methodology of the Study of Consciousness in Modern Psychophysiology. In the Book 150 years Reflexes of the Brain. Iintell., Moscow, 152 p.

[4] Yumatov, E.A. (2017) To the Theory of the Systemic Organization of the Brain Psychic Activity. Current Neurobiology, 8, 40-50.

[5] Pavlov, A.N., Hramov, A.E., Koronovskii, A.A., Sitnikova, E., Makarov, V.A. and Ovchinnikov, A.A. (2012) Wavelet Analysis in Neurodynamics. Physics-Uspekhi, 55,845 .

[6] Hramov, A.E., Koronovskii, A.A., Makarov, V.A., Pavlov, A.N. and Sitnikova, E. (2015) Wavelets in Neuroscience. Springer Heidelberg New York Dordrecht London, $318 \mathrm{p}$.

[7] Grubov, V.V., Sitnikova, E., Koronovskii, A.A., Pavlov, A.N. and Hramov, A.E. (2012) Automatic Extraction and Analysis of Oscillatory Patterns on Nonstationary EEG Signals by Means of Wavelet Transform and the Empirical Modes Method. BRAS: Physics, 76, 1361-1364.

[8] Grubov, V.V., Sitnikova, E., Pavlov, A.S., Koronovskii, A.A. and Hramov, A.E. (2017) Recognizing of Stereotypic Patterns in Epileptic EEG Using Empirical Modes and Wavelets. Physica A, 486, 206-217. https://doi.org/10.1016/j.physa.2017.05.091

[9] Nazimov, A.I., Pavlov, A.S., Nazimova, A.A., Grubov, V.V., Koronovskii, A.A., Sitnikova, E. and Hramov, A.E. (2013) Serial Identification of EEG Patterns Using Adaptive Wavelet-Based Analysis. The European Physical Journal Special Topics, 222, 2713-2722. https://doi.org/10.1140/epjst/e2013-02051-6

[10] Harris, A.M., Dux, P.E. and Mattingley, J.B. (2018) Awareness Is Related to Reduced Poststimulus Alpha Power: A Noreport Inattentional Blindness Study. European Journal of Neuroscience. https://doi.org/10.1111/ejn.13947 
[11] Koch, C., Massimini, M., Boly, M. and Tononi, G. (2016) Neural Correlates of Consciousness: Progress and Problems. Nature Reviews Neuroscience, 17, 307-321. https://doi.org/10.1038/nrn.2016.22

[12] Tsuchiya, N., Wilke, M., Frässle, S. and Lamme, V.A.F. (2015) No-Report Paradigms: Extracting the True Neural Correlates of Consciousness. Trends in Cognitive Sciences, 19, 757-770. https://doi.org/10.1016/j.tics.2015.10.002

[13] Yumatov, E.A., Potapova, O.V., Potapov, V.Y., Glazachev, O.S. and Rajewski, V.V. (2017) The Experimental Behavioral Model and Software to Identify of Mental Activity of the Brain. Psychology and Behavioral Science International Journal, 2, 1-4. https://doi.org/10.19080/PBSIJ.2017.02.555576

[14] MacDonald, M. (2012) Pro WPF 4.5 in C\#: Windows Presentation Foundation in NET 4.5. Apress, 4th Edition, 1095 p. https://doi.org/10.1007/978-1-4302-4366-3

[15] Stenning, J. (2014) Direct 3D Rendering Cookbook. Packt Publishing, 430 p.

[16] Grossman, A. and Morlet, J. (1984) Decomposition of Hardy Functions into Square Integrable Wavelets of Constant Shape. SIAM Journal on Mathematical Analysis, 15, 723-736. https://doi.org/10.1137/0515056

[17] Rey, D. and Neuhauser, M. (2011) Wilcoxon Signed-Rank Test. International Encyclopedia of Statistical Science, Springer, 1659 p.

[18] Klimesch, W. (2012) Alpha-Band Oscillations, Attention, and Controlled Access to Stored Information. Trends in Cognitive Sciences, 16, 606-617.

https://doi.org/10.1016/j.tics.2012.10.007

[19] Romei, V., Gross, J. and Thut, G. (2010) On the Role of Prestimulus Alpha Rhythms over Occipito-Parietal Areas in Visual Input Regulation: Correlation or Causation? Journal of Neuroscience, 30, 8692-8697. https://doi.org/10.1523/JNEUROSCI.0160-10.2010

[20] Mathewson, K.E., Gratton, G., Fabiani, M., Beck, D.M. and Ro, T. (2009) To See or Not to See: Prestimulus a Phase Predicts Visual Awareness. Journal of Neuroscience, 29, 2725-2732. https://doi.org/10.1523/JNEUROSCI.3963-08.2009 